\title{
Success Is All in the Measures: Androgenousness, Curvaceousness, and Starring Frequencies in Adult Media Actresses
}

\author{
Martin Voracek, D.Sc., Ph.D. ${ }^{1}$ and Maryanne L. Fisher, Ph.D. ${ }^{2,3}$
}

Received June 7, 2004; revision received September 2, 2005; accepted September 10, 2005 Published online: 24 June 2006

The debate of whether body-mass index (BMI) or waist-to-hip ratio (WHR) is the primary visual cue to female physical attractiveness (FPA) has generated considerable interest. However, experiments addressing this question typically have limited ecological validity and do not capture the ultimate goal of FPA, which is to elicit male sexual arousal. Hence, using an unobtrusive measures design, we retrieved movie and magazine starring frequencies of 125 adult media actresses from a company's database, operationalized starring frequencies as FPA measures, and tested their relationship to actresses' anthropometric data. Low BMI was related to frequent movie starring, while WHR, waistto-bust ratio (WBR), and bust size were not. Conversely, low WHR, low WBR, and larger bust size were related to frequent magazine starring, while BMI was not. Visual cues to FPA might be domain-specific, with androgenousness cues salient for attractiveness evaluation of moving bodies and curvaceousness cues salient for posing bodies.

KEY WORDS: female attractiveness; waist-to-hip ratio; body-mass index; pornography.

\section{INTRODUCTION}

Visual cues to human female physical attractiveness (FPA), and their evolutionary underpinnings, are a topic of current debate. In an influential study by Singh (1993), evidence was provided to suggest that waist-to-hip ratio (WHR) is a primary visual cue for FPA. Singh found that the WHR maximally sexually attractive to males (i.e., 0.70 or slightly lower) was strongly correlated to health-related optimums in regard to fertility and endocrinological status, as well as a lower risk for various life-threatening and chronic diseases and an increase in longevity. It was also shown that the preferred WHR was cross-culturally stable and thus a human universal. Further, it is secularly stable and preference is not subject to drift or sudden change.

\footnotetext{
${ }^{1}$ Department of Basic Psychological Research, School of Psychology, University of Vienna, Vienna, Austria.

${ }^{2}$ Department of Psychology, St. Mary’s University, Halifax, Nova Scotia, Canada.

${ }^{3}$ To whom correspondence should be addressed at Department of Psychology, St. Mary's University, 923 Robie Street, Halifax, Nova Scotia B3H 3C3, Canada; e-mail: mlfisher@smu.ca.
}

Several researchers have questioned the value range (Tassinary \& Hansen, 1998), the cross-cultural stability (Furnham \& Nordling, 1998), the limited consideration for local environments (Sugiyama, 2004), and the secular stability (Freese \& Meland, 2002; Voracek \& Fisher, 2002) of the optimally attractive female WHR. Particularly, one research group (Tovée \& Cornelissen, 2001; Tovée, Maisey, Emery, \& Cornelissen, 1999; Tovée, Reinhardt, Emery, \& Cornelissen, 1998; Tovée, Tasker, \& Benson, 2000) has gone further, marshaling evidence to suggest that body-mass index (BMI), or weight scaled for height $\left(\mathrm{kg} / \mathrm{m}^{2}\right)$, is a more salient determinant of FPA than WHR. They argued that previous findings on WHR were artifactual because the stimuli used to examine WHR variation were confounded with BMI variation. It is of note that, similar to WHR, BMI tends to increase with age and temporally changes during pregnancy. More generally, like WHR, BMI is regarded as a honest visual signal that advertises health status, nutritional condition, physical prowess, and the underlying endocrine configuration (Fink, Neave, \& Manning, 2003).

It has also been argued that, in general, research on FPA continually relies on weak methodologies (Henss, 
2000) or is of low ecological validity, relative to facial attractiveness research. Ecological validity is an important consideration, as it is the extent to which research findings are generalizable across settings or environmental conditions, or most importantly, from experimental conditions to differing environments. Therefore, ecological validity reflects the extent to which observed behavior in the laboratory is generalizable to behavior in natural situations. A cursory view of stimulus quality and stimulus evaluation procedures, as currently employed in this line of research, supports the above observation. For instance, to retain consistency with previous work, Singh (1993) employed cartoon-like drawings of female silhouettes wearing swimsuits that were devoid of primary and secondary sexual characteristics. Tovée and Cornelissen (2001), (Tovée et al., 1998, 1999, 2000), as well as other researchers, used unnatural standardized photographs of posed females with face and hair masked, arms extended, wearing grey tight clothing, and so forth (see Thornhill \& Grammer, 1999). With the exception of the Thornhill and Grammer (1999) study, full-body photographs of naked females have not been used. Stimuli with anatomical detailing of primary and secondary sexual characteristics have only rarely been tried (Furnham \& Nordling, 1998). Further, no study has included the evaluation of moving, naked female bodies.

Likewise, the stimulus evaluation procedures in FPA research are of questionable generalizability. Typically, evaluations consist of Likert-scale ratings for course credit that are made mainly by male undergraduates and, in one case, solely by female undergraduates (Forestell, Humphrey, \& Stewart, 2004). With the exception of a few accounts (Freese \& Meland, 2002; Singh, 1993; Tovée, Mason, Emery, McClusky, \& Cohen-Tovée, 1997; Voracek \& Fisher, 2002), where body measures of fashion models or Playboy centerfolds were investigated, there has been a lack of FPA studies using unobtrusive measures (Webb, Campbell, Schwartz, \& Sechrest, 2000).

We argue that, ultimately, FPA has been evolutionarily designed to elicit explicit sexual behavior from males (see also Berry, 2000). In other words, physical attractiveness is by implication sexual attractiveness. This ultimate goal of FPA has not been adequately captured by any of the aforementioned studies. In accordance with Malamuth (1996) and Salmon and Symons (2001), we posit that male-oriented pornography is a valid way for capturing this ultimate goal of attractiveness. Adult media's "characteristic features have been shaped in free markets by the cumulative choices made by ... men ... who have 'voted' with their money" (Salmon \& Symons, 2001, p. 56). In addition, adult media contain "minimal plot development, focusing instead on the sex acts themselves and emphasizing the display of female bodies" (p. 59). Finally, "women porn stars manifest cues of high mate value in that they are young and physically attractive" (p. 60). In short, "the imagery of pornography tends to correspond to the preexisting fantasy imagery of the consumer.... Thus, the prevalence of a specific type of imagery in pornography is an unobtrusive measure of the prevalence of the corresponding fantasy among the consumers" (Dietz \& Evans, 1982, p. 1493). This ecological validity is also supported by the facts that sexual fantasy is by far the most common form of human sexual experience (Ellis \& Symons, 1990). Furthermore, men's sexual fantasies are generally more sexually explicit than women's sexual fantasies and tend to focus on particular female body parts (for a review, see Okami \& Shackelford, 2001).

In previous research on FPA, primarily male study participants rated the attractiveness of line drawings or photographs, with WHR and/or BMI varied across stimuli. To overcome the weak ecological validity of this approach, we used unobtrusive measures in the present study and looked in vivo at stimuli men want, are inclined to spend money for, and find sexually arousing. This study used adult media actresses' ("porn stars") body measures as stimuli and we operationally defined actresses' starring frequencies as FPA measures. Employing these measures, we addressed the question of whether BMI or WHR, both pitted against other body measures, is a more salient visual cue of FPA.

\section{METHOD}

\section{Subjects}

The source and nature of the data were as follows. We used data from an established leading European adult media company (Private Media Group Inc., see www.private.com) that is listed on the Nasdaq stock market and has business activities in over 35 countries around the world. The company's websites reportedly attract around 25 million visitors a month, totaling approximately 650 million page viewings per month. The company has a free-access online shop with product lists and information, including a model database comprised of (as of mid-2001) 1,084 entries of female models starring in at least one company product (magazine or movie) since the early 1990s. The database can be searched by both model name and product, such that all starrings for each model can be obtained. The movie and the magazine products depict heterosexual activities and the magazine 
contents (pictorials of sexual acts) can be regarded as equally sexually explicit as the movie contents.

In another online area of the company that is payfor-access, key anthropometric and biographic data, stills, character profiles, and, occasionally, interviews for 125 (as of mid-2001) selected models out of the total 1,084 are provided. We hereafter refer to this premium group of 125 selected models as the "elite" models, and to the remainder (959 models), using the company's term, as the "talents." Anthropometric data for the talents group were not available and are therefore not included in analysis.

\section{Procedure}

For the elite models, we extracted eight variables from the online database: information on height and weight; bust, waist, and hip size; and birth date, country of origin, and ethnicity. We also did a validity check of the grouping done by the company into adult media talents versus elite actresses. The company's distinction appeared not to be arbitrary, as the talents group $(N=959)$ accounted for a total of 545 magazine starrings (pictorials) and 1,465 movie starrings, whereas the much smaller $(N=125)$ elite models group accounted for 310 magazine and 752 movie starrings (see Table I for descriptive statistics). Elite models had markedly higher numbers of magazine starrings $(z=11.12 ; p<.001)$ and movie starrings $(z=14.24 ; p<.001)$ relative to the talents group (Mann-Whitney $U$ test).

\section{Measures}

We considered the adult media actresses' body measures as unobtrusive attractiveness stimuli. From the anthropometric data, we calculated the following composite body shape measures: body-mass index (BMI), which is weight scaled for height or degree of "tubular" body shape; waist-to-hip ratio (WHR), which is degree of lower-body curvaceous shape; waist-to-bust-ratio (WBR),

Table I. Descriptive Statistics of Adult Media Elite Actresses' $(N=125)$ versus Talent Actresses' $(N=959)$ Starring Frequencies

\begin{tabular}{lcccc}
\hline Actress group and starring type & $M$ & Median & $S D$ & Range \\
\hline Elite actresses (MOSF) & 6.02 & 3 & 4.78 & $0-31$ \\
Talent actresses (MOSF) & 1.53 & 1 & 1.94 & $0-15$ \\
Elite actresses (MASF) & 2.48 & 2 & 2.58 & $0-17$ \\
Talent actresses (MASF) & 0.57 & 0 & 0.88 & $0-6$ \\
\hline
\end{tabular}

Note. MOSF, Movie starring frequency; MASF, Magazine starring frequency. which is degree of upper-body curvaceous shape; and bust-to-hip ratio (BHR), which is degree of "hourglass" body shape (Tovée et al., 1997). BMI is a bodily cue of androgenousness (Tovée et al., 1997, 1998, 1999), where androgeny indicates that the body shape is not overtly feminine nor masculine. In contrast, WHR, WBR, and BHR are cues of curvaceousness (Tovée et al., 1997, 1998, 1999), meaning the level of gentle or rounded angularity that is evident in body shape. From the models' birth date, we calculated models' age (as of mid-2001). Descriptive statistics of single and composite body measures for the 125 elite models are presented in Table II. Most of the models were Caucasian (92.0\%), from Europe (84.8\%), with a sizeable portion from the post-Soviet transitional countries of Eastern Europe (38.4\%).

Further, we considered the adult media actresses' starring frequencies as unobtrusive attractiveness measures. Individual magazine starring frequencies (MASF) and movie starring frequencies (MOSF) from the online data source were obtained for each of the elite and talent models. Since we did not weight or rate the starring frequencies in any manner, these count variables were only proxy attractiveness measures with presumable, but not quantifiable, imperfect reliability. On the other hand, since starring frequency is an outcome that is shaped by a self-regulating international market, it is an ecologically valid unobtrusive measure of models' attractiveness and consequent success.

\section{RESULTS}

\section{Relations of Adult Media Actresses' Body Measures and Starring Frequencies}

Correlations between elite models' single and composite body measures, as well as age, with MOSF and MASF are given in Table II (all $d f \mathrm{~s}=123$ ). Models' MOSF was significantly negatively related to models' BMI $(r=-.176 ; p=.049)$, but was not related to models' bust size, WBR, or WHR. Conversely, models' MASF was significantly negatively related to models' WBR $(r=-.219 ; p=.014)$, significantly positively related to bust size $(r=.185 ; p=.039)$, and negatively related to WHR, although not statistically significant $(r=-.132)$, but not related to BMI. No other single or composite body measures showed significant association with MOSF or MASF. Also, models' age was not related to cumulative starring frequencies, and thus time spacing of starrings must be highly variable across models.

Interestingly, WHR and BMI varied almost independently in our sample $(r=.051)$. As well, WHR was 
Table II. Descriptive Statistics of Adult Media Elite Actresses' Body Measures and Age, and Relations with Actresses' Starring Frequencies $(N=125)$

\begin{tabular}{lcccccc}
\hline \multicolumn{1}{c}{ Variables } & $M$ & Median & $S D$ & Range & $r$ (MOSF) & $r$ (MASF) \\
\hline Bust (cm) & 85.8 & 86 & 6.0 & $70-97$ & -.001 & $.185^{*}$ \\
Hip (cm) & 87.4 & 89 & 4.8 & $74-96$ & -.083 & .103 \\
Waist (cm) & 59.9 & 60 & 3.7 & $45-76$ & -.089 & -.040 \\
Height (cm) & 168.5 & 168 & 4.8 & $156-183$ & .070 & .065 \\
Weight (kg) & 52.3 & 52 & 4.4 & $43-73$ & -.109 & .020 \\
Body-mass index & 18.4 & 18.3 & 1.3 & $15.2-22.5$ & $-.176^{*}$ & -.027 \\
Waist-to-hip ratio & 0.68 & 0.67 & 0.04 & $0.60-0.88$ & -.013 & -.132 \\
Waist-to-bust ratio & 0.70 & 0.69 & 0.05 & $0.60-0.86$ & -.066 & $-.219^{*}$ \\
Bust-to-hip ratio & 0.98 & 0.99 & 0.05 & $0.82-1.10$ & .081 & .133 \\
Age (years) & 24.3 & 23.8 & 2.8 & $19.5-31.8$ & -.013 & -.091 \\
\hline
\end{tabular}

Note. MOSF; Movie starring frequency, MASF; Magazine starring frequency.

${ }^{*} p<.05 ; d f=123$.

significantly negatively related with age $(r=-.207$; $p=.021)$, but BMI was not $(r=.018)$. BMI was significantly negatively correlated with height $(r=-.188$; $p=.036$ ) and positively correlated, in a highly significant manner, with weight $(r=.727 ; p<.001)$ in the present sample.

\section{Corroborating the Relations of Body Measures and Starring Frequencies: Higher-Order Partial Correlations}

Control of potential confounds is crucial for the corroboration of correlational evidence. Following frequently employed procedures in previous research (cf. Thornhill \& Grammer, 1999), we conducted partial correlation analyses to determine whether the pattern of correlations would remain intact when other salient variables were statistically held constant. The pattern of the eight associations reported above (BMI, WHR, WBR, and bust size with MOSF and MASF, respectively) was largely preserved when we partialed out a number of salient variables, including competitive variables (WHR for BMI, and vice versa) and counterpart variables (MOSF for MASF, and vice versa). Models' age was partialed out from all the correlations. The evidence from the higher-order partial correlation analyses is reported in the remainder of this section (all $d f \mathrm{~s}=119$ ).

First, the significant association between BMI and $\operatorname{MOSF}(r=-.176 ; p=.049)$ remained when controlled for models' age, bust size, WHR, and $\operatorname{MASF}\left(r_{\mathrm{p}}=-.185\right.$; $p=.043)$. Likewise, the nonassociation between BMI and $\operatorname{MASF}(r=-.027)$ did not change to a significant relation when controlled for models' age, bust size, WHR, and $\operatorname{MOSF}\left(r_{\mathrm{p}}=.074\right)$.
Second, the negative association between WHR and MASF $(r=-.132)$ remained (achieving marginal significance) when controlled for models' age, bust size, BMI, and MOSF $\left(r_{\mathrm{p}}=-.169 ; p=.063\right)$. Similarly, the nonassociation between WHR and MOSF $(r=-.013)$ did not change to a significant relation when controlled for models' age, bust size, BMI, and MASF $\left(r_{\mathrm{p}}=.116\right)$.

Third, the significant association between bust size and $\operatorname{MASF}(r=.185 ; p=.039)$ remained when controlled for models' age, BMI, WHR, and MOSF $\left(r_{\mathrm{p}}=.192\right.$; $p=.035)$. Likewise, the nonassociation between bust size and MOSF $(r=-.001)$ did not change to a significant relation when controlled for models' age, BMI, WHR, and $\operatorname{MASF}\left(r_{\mathrm{p}}=-.093\right)$.

Lastly, the significant association between WBR and MASF $(r=-.219 ; p=.014)$ remained when controlled for models' age, hip size, BMI, and MOSF $\left(r_{\mathrm{p}}=-.199\right.$; $p=.029)$. Similarly, the non-association between WBR and MOSF $(r=-.066)$ did not change to a significant relation when controlled for models' age, hip size, BMI, and $\operatorname{MASF}\left(r_{\mathrm{p}}=.061\right)$.

This stability of the correlational pattern adds credence to the findings and is particularly striking since MOSF and MASF were highly positively correlated in the sample $(r=.656 ; p<.001)$. Since the relations between bodily measures with one of the starring frequency variables largely remained when the other starring frequency was held constant, MOSF and MASF likely measure different "facets" of model success.

\section{DISCUSSION}

We have shown a differential pattern of relations between several bodily measures and starring frequencies 
in a sample of contemporary adult media actresses. In adult media, it appears that slimmer or less curvaceous models are selected to star in movies, whereas more busty or curvaceous models more frequently star in magazines. An alternative to this market-driven hypothesis, namely the possibility of self-selection of models to either movie or magazine starring according to variation in models' androgen levels, was not testable within this study but is, in our view, rather unlikely.

It is of note that WHR and BMI varied almost independently in our sample of adult media actresses. Previous experimental research has clearly confounded WHR with BMI, and it has been claimed that the two are highly correlated in women (Puhl \& Boland, 2001). We also note that WHR was significantly negatively related with age, but BMI was not. Longitudinally, a negative relation between WHR and age would be an unexpected finding (Singh, 1993). Because we analyzed cross-sectional data, the negative relation is suggestive of a cohort effect such that later born actresses had a higher WHR than earlier born actresses. This finding is in line with a recent trend of a WHR increase observed in Playboy centerfolds (Freese \& Meland, 2002; Voracek $\&$ Fisher, 2002). While previous reports found BMI independent from height and strongly related to weight in the female general population (Manson et al., 1995), BMI was significantly negatively correlated with height and strongly positively correlated with weight in the present sample.

How do the findings of the present study converge with evidence from previous research on FPA? What is their relevance for attractiveness research? We address these issues in the following sections.

\section{Body-Mass Index and Waist-To-Hip Ratio as Domain-Specific Attractiveness Cues}

First of all, posing the question of whether there is one primary visual cue of FPA (be it either WHR or BMI), as investigated in various recent research accounts, seems mistaken and overly simplified. This either/or question does not acknowledge that there are different domains of attractiveness evaluation, such as with still postures versus movement. The domain of movement and gait is ecologically more valid, relative to the domain of postures, simply because people encountered are in movement. It seems that the salience of WHR as a prime attractiveness criterion is largely an artifact from the typical stimuli used in previous research (i.e., line drawings or still photographs) and that WHR progressively becomes less salient as stimuli become more ecologically valid.
Recent research provides evidence for this contention. Tovée and Cornelissen (2001) had participants rate the attractiveness of color images of real women in a frontal-view and in a side-view. They found that BMI explained approximately 30 times more variance in attractiveness ratings than WHR, suggesting that BMI was a considerably stronger determinant of FPA than WHR. Further, they found BMI had a high view invariance, whereas WHR was less view-invariant, such that the anatomical constituents of WHR were more obvious in profile, and changing the viewing angle also changed their visual salience. According to Tovée and Cornelissen, the difficulty in accurately judging WHR must have consequences on its evolutionary significance: "If a physical feature has only a comparatively weak visual proxy, then it does not matter how good a predictor it is of health and fertility, it will play only a secondary role in sexual selection. This may be true of WHR and could explain why it plays a subsidiary role to BMI, which has a more reliable visual proxy" (Tovée \& Cornelissen, 2001, p. 400). Relatedly, Thornhill and Grammer (1999) found that attractiveness ratings of male judges of three picture types-facial, front, and back photographs across the same set of nude females-were more highly correlated with BMI than with WHR.

If it is difficult to accurately judge females' WHR from differently angled still photographs, it must be even more difficult to judge females' WHR from movie sequences. True variance in WHR might be too small and WHR view invariance too large to get an accurate impression when a moving body is observed. This difference in variance may be the reason why BMI has dominance in the overall impression and attractiveness evaluation of moving bodies.

The findings of the present study, as well as those of Tovée and Cornelissen (2001) and Thornhill and Grammer (1999), cast doubt on Singh's (1993) “firstpass filter" theory that states one role for WHR is that of a distant attractiveness evaluation category. This theory could actually be applied more strongly for BMI. From a far distance, upon approaching a female silhouette, one is likely to first perceive the width of the body (as largely captured by BMI), and afterwards details such as the curvaceousness (as captured by WHR and also by WBR).

Less salience for WHR as an attractiveness criterion in the domain of moving bodies is also evident from the findings of Lawson, Tassinary, and Johnson (1999). Lawson et al. constructed animated human 3D silhouettes in which physical sexual features were omitted and systematically varied WHR with twist index (TI) of gait. They crossed five WHR values $(0.5-0.9)$ with five TI values (from feminine hip "sway" to masculine shoulder 
"swagger") and presented the 25 distinct walking figures on a monitor to male and female raters. Overall, the walking silhouette judged to be the most physically attractive had a WHR of 0.5 (superfeminine), coupled with a superfeminine walk motion pattern (pronounced hip "sway"). The results of this study also indicated that judgments of sex and physical attractiveness relied heavily on both physical proportion and motion cues. In particular, WHR seemed to primarily act as a cue for raters' judgments of sex. Although not addressed in the Lawson et al. (1999) experiment, we suggest that women with low BMI more likely have a superfeminine gait and a movement pattern of youthful appearance-compare "catwalking" fashion models - than higher BMI women. This association could be a prime reason for our finding that adult media actresses with lower BMI had a higher MOSF.

On the other hand, the relation of curvaceousness cues and MASF in our data set was in line with previous findings (Singh, 1993) regarding the salience of WHR as a cue for FPA when evaluated from nonmoving images. However, the relation between androgenousness cues and MOSF in our data was concordant with the recent findings that WHR loses salience, relative to BMI, when the stimulus material becomes more ecologically valid, such as the evaluation of moving rather than still bodies (Lawson et al., 1999).

In essence, adult magazine pictorials can be regarded as 2D-like stimulus material since they are still, whereas adult movie scenes are 3D-like stimulus material, as they depict bodies in motion. The differential relationship of androgenousness and curvaceousness cues with unobtrusive FPA measures in the present study was further supported by documented differences in body measures of glamour models versus fashion models (see comparative data in Tovée et al., 1997). Glamour models, who typically pose in magazines, have more curvaceous body shapes, whereas fashion models are frequently seen in motion, such as on the catwalk, and have more androgynous body shapes. Obviously, the way in which images are represented markedly affects perception and evaluation, with the less information provided the more exaggeration permitted. The 1930s cartoon character Betty Boop is sexy as a line drawing, but not so if her form would come to life (D. Symons, personal communication, April 10, 2002).

\section{Implications of Male Preference for Low Body-Mass Index Females}

What could be the evolutionary significance of a low BMI female shape? While it is true that cross-cultural differences in weight and shape ideals can be observed (Anderson, Crawford, Nadeau, \& Lindberg, 1992), these appear to vary in an environmentally contingent fashion, rather than arbitrarily. Weight and shape standards appear to fluctuate within the same culture over time (Mazur, 1986). Still, there are some particular features that are either recurrent or invariant across cultures and time. Such a prime criterion for FPA that could explain our finding of differential relations is firmness of body tissue. Firmness is an important aspect of youthful appearance and is cross-culturally a salient physical mate value criterion (Buss, 1989). Firmness of tissue is related to good muscle tone, provides a visual cue for female nubility, and thus indicates current fertility and future reproductive value (Symons, 1995). Similarly, a positive relation of fluctuating asymmetry (a pointer to phenotypic quality) and female body weight, such that higher symmetry is related to lower body weight, has been reported (Manning, 1995). There is also evidence that bodily androgenousness is a reliable cue of female reproductive value. For instance, Kirchengast and Winkler (1996), in studying tribal !Kung San and Kavango people of Namibia, found that lighter weight females had significantly more births, and a greater number of surviving offspring, than overweight females. Likewise, and contrary to widely held beliefs, women from industrialized nations presenting BMI values of 20 or slightly below appear to conceive equally easily compared with women with moderate BMI values (20-25) and markedly more easily than those who are overweight (Ryley et al., 2004). Further, there is evidence from Western cultures that, relative to low BMI females, heavier women suffer far more frequently from a severe decrease in postmenopausal sexual interest (Kirchengast \& Winkler, 1996). High female BMI might be a signal for a lower sexual drive and possibly for an earlier onset of menopause, leading to lower fertility and reproductive success. Conversely, low BMI women who show associated signs of nubility, such as tissue firmness, sexy gait, and gracious movements, may signal a higher sexual drive which possibly leads to higher reproductive success. Thus, a male preference for light-weight women may well be adaptive. Ancestrally, there must have been low variation in BMI, since becoming overweight seems unlikely in those environments. We are aware that there is some controversy in the literature over whether BMI and WHR actually are sexually selected traits or better conceived as consequences of other selection pressures (cf. Anderson, 1988; Pawlowski \& Grabarczyk, 2003), but this question must remain unsettled for now, as our findings do not add meaningfully to this debate.

On average, a low WHR body has wider hips and larger buttocks and may look optimal when not 
moving, but less than optimal when moving because the tissue is, due to these features, not maximally firm. In contrast, a low BMI body may look optimally attractive in motion, because gracious movements reveal that the tissue is maximally firm, but may look less attractive when not moving (i.e., too tubular). Mapped onto adult media products, it is possible to make female models with nonoptimal tissue firmness look attractive in still pictures as the photographer can adjust angle and pose, etc., whereas in movies, nonoptimally firm tissue and less gracious movements due to heavier weight and curvaceousness cannot be disguised. Low BMI womenin adult media products, on the catwalk, when dancing, or elsewhere-may possibly compensate for a lack of curvaceousness by moving their bodies in such a way as to create the illusion of more shape, and, by extension of argument, may be able to get into different poses with more ease and grace than curvier women. Furthermore, low BMI could be related to other traits salient for adult media starring, such as facial and vocal expressivity, sexual skills, or acting ability, which could mediate the observed link between low BMI and frequent movie starring. Both of these hypotheses are testable.

\section{Future Avenues for Physical Attractiveness Research}

Three concluding conceptual thoughts can be derived from the findings of this study. First, analyzing commercial erotica is a worthwhile avenue for studying aspects of human physical attractiveness, especially from an evolutionary standpoint. Male sexual arousal in response to pornography is an obvious by-product of the evolved adaptation of male sexual arousal to young nude women (Okami \& Shackelford, 2001). Relatedly, Malamuth (1996) has noted an "uncanny correspondence between the major adaptive problems defined by evolutionary psychologists and the content of sexually explicit media" (p. 20). Thus, researchers should "test the prediction that the type of physical attributes recurrently featured in sexually explicit media are likely to have been associated with reproductive success in ancestral environments." Specifically, Malamuth proposed the testing of the prediction "that a relatively high degree of body symmetry will be found in the models chosen for such portrayals." This is an obvious next step. We predict that for adult media actresses a higher degree of body symmetry will be associated with higher starring frequencies.

Second, future investigations should examine the replicability of the findings from this unobtrusive measures design, along with the hypotheses derived from the findings of Tovée and Cornelissen (2001) and Lawson et al. (1999) in an experimental design. A future experiment should test in a controlled manner whether curvaceousness cues like WHR progressively lose salience, relative to androgenousness cues like BMI, in attractiveness ratings of female bodies that have a progressive range from ecologically less valid to ecologically valid. We predict that in such an experiment domain-specificity of bodily cues of curvaceousness versus androgenousness with regard to the domains of stills and postures (2Dlike stimuli) versus animations and movements (3D-like stimuli) will replicate. If so, domain-specificity of FPA cues could have the potential to reconcile the opposing evidence of previous BMI and WHR research.

Third, the findings of the present study emphasize the necessity to substantially broaden the approach of physical attractiveness research, as has been concluded in a recent major review (Berry, 2000). A shortcoming of virtually all research in this area is that single, visually perceived body cues that are static in nature, such as the predominant use of WHR, have been extensively employed as FPA measures. Physical attractiveness extends far beyond WHR, and is better conceptualized as dynamic and multimodal. Dynamic information, such as an individual's gait pattern and other body movement style characteristics, is not only a reliable cue to sex, age, psychological and physiological health, but also influences how sexually attractive one is perceived. Consequently, attractiveness research has to move beyond mere morphological variables and must include dynamic information. Grammer, Honda, Juette, and Schmitt (1999) have introduced a promising technique, dubbed motion energy detection, which is the automatic analysis of changes of body contours from digitized films. This technique yields a quantitative assessment of body movement qualities, such as speed, duration, size, and other parameters, independent of the movements' gestalt and form. We anticipate that the motion energy detection technique will unveil notable differences in movement quality among females, depending on values and combinations of prime cues of androgenousness and curvaceousness. Specifically, we predict that for the data set we examined, there are distinct differences in movement quality between the elite models group and the talents group, as well as differences in movement quality between the highest-ranking elite models, as evaluated from the starring frequencies, and others who have starred less frequently.

Our study provides evidence of a pattern of differential relations between androgenousness versus curvaceousness cues with that of magazine versus movie starring frequencies by adult media actresses. This pattern has been explored within an evolutionary framework of 
sexual selection and associated male mating psychology. Further, it possibly has the potential to reconcile opposing findings of previous BMI and WHR research. Visual cues to FPA might be domain-specific, with bodily androgenousness cues being salient for attractiveness evaluation of moving female bodies, and curvaceousness cues being salient for posing female bodies.

\section{REFERENCES}

Anderson, J. L. (1988). Breast, hips, and buttocks revisited: Honest fatness for honest fitness. Ethology and Sociobiology, 9, 319324.

Anderson, J. L., Crawford, C. B., Nadeau, J., \& Lindberg, T. (1992). Was the Duchess of Windsor right? A cross-cultural review of the socioecology of ideals of female body shape. Ethology and Sociobiology, 13, 197-227.

Berry, D. S. (2000). Attractiveness, attraction, and sexual selection: Evolutionary perspectives on the form and function of physical attractiveness. Advances in Experimental Social Psychology, 32, 273-342.

Buss, D. M. (1989). Sex differences in human mate preferences: Evolutionary hypotheses tested in 37 cultures. Behavioral and Brain Sciences, 12, 1-49.

Dietz, P. E., \& Evans, B. (1982). Pornographic imagery and prevalence of paraphilia. American Journal of Psychiatry, 139, 1493-1495.

Ellis, B. J., \& Symons, D. (1990). Sex differences in sexual fantasy: An evolutionary psychological approach. Journal of Sex Research, 27, 527-556.

Fink, B., Neave, N., \& Manning, J. T. (2003). Second to fourth digit ratio, body mass index, waist-to-hip ratio, and waist-to-chest ratio: Their relationships in heterosexual men and women. Annals of Human Biology, 30, 728-738.

Forestell, C. A., Humphrey, T. M., \& Stewart, S. H. (2004). Involvement of body weight and shape factors in ratings of attractiveness by women: A replication and extension of Tassinary and Hansen (1998). Personality and Individual Differences, 36, 295-305.

Freese, J., \& Meland, S. (2002). Seven tenths incorrect: Heterogeneity and change in the waist-to-hip ratios of Playboy centerfold models and Miss America pageant winners. Journal of Sex Research, 39, 133-138.

Furnham, A., \& Nordling, R. (1998). Cross-cultural differences in preferences for specific male and female body shapes. Personality and Individual Differences, 25, 635-648.

Grammer, K., Honda, M., Juette, A., \& Schmitt, A. (1999). Fuzziness of nonverbal courtship communication unblurred by motion energy detection. Journal of Personality and Social Psychology, 77, 487508 .

Henss, R. (2000). Waist-to-hip ratio and female attractiveness: Evidence from photographic stimuli and methodological considerations. Personality and Individual Differences, 28, 501-513.

Kirchengast, S., \& Winkler, E. M. (1996). Nutritional status as indicator for reproductive success in !Kung San and Kavango females from Namibia. Anthropologischer Anzeiger, 54, 267-276.

Lawson, K. D., Tassinary, L. G., \& Johnson, S. P. (1999). Psychophysiology and the "suspension of disbelief" [Abstract]. Psychophysiology, 36(Suppl. 1), S72.
Malamuth, N. M. (1996). Sexually explicit media, gender differences, and evolutionary theory. Journal of Communication, 46, 8-31.

Manning, J. T. (1995). Fluctuating asymmetry and body weight in men and women: Implications for sexual selection. Ethology and Sociobiology, 16, 145-153.

Manson, J. E., Willet, W. C., Stampfer, M. J., Colditz, G. A., Hunter, D. J., Hankinson, S. E., et al. (1995). Body weight and mortality among women. New England Journal of Medicine, 333, 677-685.

Mazur, A. (1986). U.S. trends in feminine beauty and overadaptation. Journal of Sex Research, 22, 281-303.

Okami, P., \& Shackelford, T. K. (2001). Human sex differences in sexual psychology and behavior. Annual Review of Sex Research, 12 , 186-241.

Pawlowski, B., \& Grabarczyk, M. (2003). Center of body mass and the evolution of female body shape. American Journal of Human Biology, 15, 144-150.

Puhl, R. M., \& Boland, F. J. (2001). Predicting female physical attractiveness: Waist-to-hip ratio versus thinness. Psychology, Evolution and Gender, 3, 27-46.

Ryley, D. A., Bayer, S. R., Eaton, J., Zimon, A., Klipstein, S., \& Reindollar, R. (2004). Influence of body mass index (BMI) on the outcome of 6827 IVF cycles [abstract]. Fertility and Sterility, 82(Suppl. 2), S38-S39.

Salmon, C., \& Symons, D. (2001). Warrior lovers: Erotic fiction, evolution and female sexuality. London: Weidenfeld \& Nicolson.

Singh, D. (1993). Adaptive significance of female physical attractiveness: Role of waist-to-hip ratio. Journal of Personality and Social Psychology, 65, 293-307.

Sugiyama, L. S. (2004). Is beauty in the context-sensitive adaptations of the beholder? Shiwiar use of waist-to-hip ratio in assessments of female mate value. Evolution and Human Behavior, 25, 51-62.

Symons, D. (1995). Beauty is in the adaptations of the beholder: The evolutionary psychology of human female sexual attractiveness. In P. R. Abramson \& S. D. Pinkerton (Eds.), Sexual nature, sexual culture (pp. 80-118). Chicago: University of Chicago Press.

Tassinary, L. G., \& Hansen, K. A. (1998). A critical test of the waist-to-hip-ratio hypothesis of female physical attractiveness. Psychological Science, 9, 150-155.

Thornhill, R., \& Grammer, K. (1999). The body and face of woman: One ornament that signals quality? Evolution and Human Behavior, 20, $105-120$.

Tovée, M. J., \& Cornelissen, P. L. (2001). Female and male perceptions of female physical attractiveness in front-view and profile. British Journal of Psychology, 92, 391-402.

Tovée, M. J., Maisey, D. S., Emery, J. L., \& Cornelissen, P. L. (1999). Visual cues to female physical attractiveness. Proceedings of the Royal Society of London Series B 266, 211-218.

Tovée, M. J., Mason, S., Emery, J. L., McClusky, S. E., \& Cohen-Tovée, E. M. (1997). Supermodels: Stick insects or hourglasses? Lancet, 350, 1474-1475.

Tovée, M. J., Reinhardt, S., Emery, J. L., \& Cornelissen, P. L. (1998). Optimum body-mass index and maximum sexual attractiveness. Lancet, 352, 548.

Tovée, M. J., Tasker, K., \& Benson, P. J. (2000). Is symmetry a visual cue to attractiveness in the human female body? Evolution and Human Behavior, 21, 191-200.

Voracek, M., \& Fisher, M. L. (2002). Shapely centrefolds? Temporal change in body measures: Trend analysis. British Medical Journal, $325,1447-1448$.

Webb, E. J., Campbell, D. T., Schwartz, R. D., \& Sechrest, L. (2000). Unobtrusive measures (Rev. ed.). Thousand Oaks, CA: Sage Publications. 